\title{
The Role of Acupoint Application of Herbal Medicine for Asthma: Meta-Analysis of Randomized Double-Blind Placebo-Controlled Trials
}

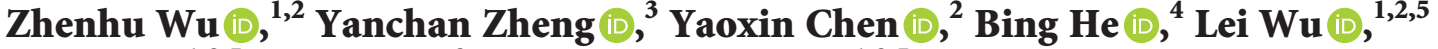 \\ Lin Lin $\mathbb{D}^{1},{ }^{1,2,5}$ Yinji Xu $\mathbb{D}^{2},{ }^{2}$ and Yuanbin Chen $\mathbb{D D}^{1,2,5}$ \\ ${ }^{1}$ State Key Laboratory of Dampness Syndrome of Chinese Medicine, \\ The Second Affiliated Hospital of Guangzhou University of Chinese Medicine, Guangzhou 510120, China \\ ${ }^{2}$ Department of Respiratory Medicine, \\ The Second Clinical College of Guangzhou University of Chinese Medicine and Guangdong Provincial Hospital of Chinese \\ Medicine, Guangzhou 510120, China \\ ${ }^{3}$ Department of Respiratory Medicine, Shantou Hospital of Traditional Chinese Medicine, Shantou 515000, China \\ ${ }^{4}$ Clinical Laboratory, South China Normal University Hospital, Guangzhou 510630, China \\ ${ }^{5}$ Guangdong-Hong Kong-Macau Joint Lab on Chinese Medicine and Immune Disease Research, \\ Guangzhou University of Chinese Medicine, Guangzhou, China
}

Correspondence should be addressed to Yinji Xu; xuyinji@163.com and Yuanbin Chen; chenyuanbin110@163.com

Received 19 February 2021; Revised 4 November 2021; Accepted 3 January 2022; Published 31 January 2022

Academic Editor: Guoqiang Xing

Copyright (C) 2022 Zhenhu Wu et al. This is an open access article distributed under the Creative Commons Attribution License, which permits unrestricted use, distribution, and reproduction in any medium, provided the original work is properly cited.

\begin{abstract}
Background. Acupoint application of herbal medicine (AAHM) has been widely used in China. At present, there is no systematic review of AAHM versus placebo in the treatment of asthma. This systematic review aims to assess the efficacy of AAHM for asthma. Methods. Searches were conducted in five English databases and four Chinese databases from their inceptions until December 2020. Randomized double-blind placebo-controlled trials were screened, and included studies evaluated routine pharmacotherapy (RP) plus AAHM versus RP plus placebo or AAHM versus placebo. The Cochrane risk of bias tool and Grading of Recommendations Assessment, Development and Evaluation (GRADE) were performed to evaluate the methodological quality and quality of evidence separately. Results. Sixteen studies involving 1,730 participants were included in this review. Compared with placebo plus RP, participants receiving long-term AAHM plus RP showed improvement in asthma quality of life questionnaire (AQLQ) with moderate-quality evidence (MD 6.53 points, 95\% CI 2.70 to 10.36). Low-quality evidence indicated that AAHM plus RP was associated with improved $\mathrm{FEV}_{1}(\%)$ compared with placebo plus RP, whether long- or short-term use (MD $11.80 \%$, 95\% CI 2.84 to 20.76; MD 10.57\%, 95\% CI 8.40 to 12.74 ; respectively). Moderate-quality evidence showed that participants receiving short-term AAHM were associated with a higher AQLQ score (MD 6.57 points, 95\% CI 3.76 to 9.38) and a lower frequency of acute exacerbations (MD -1.84, 95\% CI -2.32 to -1.36) compared with placebo. Low-quality evidence also indicated that AAHM was associated with improved $\mathrm{FEV}_{1}(\mathrm{~L})$ compared with placebo, whether long- or short-term use (MD 0.35 litres, $95 \%$ CI 0.03 to 0.67 ; MD 0.66 litres, $95 \%$ CI 0.59 to 0.73 ; respectively). Conclusions. Moderate-quality evidence is promising that AAHM can improve the quality of life and reduce acute exacerbations in patients with asthma. AAHM also shows a positive role in improving lung function, but the evidence is so indefinite due to low quality.
\end{abstract}

\section{Introduction}

With the rapid advance of urbanization and industrialization leading to air pollution and lifestyle changes, the global incidence of asthma has increased. Currently, there are about 300 million people with asthma, and the number may reach 400 million by 2025 [1]. Asthma is not only a serious threat to human health but also a cause of enormous social 
and economic burden $[2,3]$. Acupoint application of herbal medicine (AAHM) is a commonly used traditional Chinese medicine (TCM) technique for asthma [4]. AAHM is based on TCM meridian theory and involves the application of herbal paste on specific acupuncture points (also called point sticking therapy and acupoint plaster therapy). To make the paste, herbal medicines with antiasthmatic properties are ground into powder; reconciled into a paste; made into an ointment, pill, or pie; and then directly applied to acupoints. The paste is left on the acupoints for several hours, and then a new paste is applied every few days for weeks or months.

International conventional medicine guidelines recommend inhaled corticosteroids (ICS) for the long-term treatment of asthma [5]. However, long-term use can produce side effects such as increased fracture risk, osteoporosis, or oropharyngeal candidiasis [6]. Moreover, cessation of ICS after long-term use can cause symptom recurrence and increased risk of airway inflammation. Therefore, complementary and alternative therapies, including acupoint application, may be useful for the management of asthma.

Preclinical studies have demonstrated that AAHM can improve immunity, correct the TH1/TH2 proportional imbalance, and prevent the occurrence of airway inflammation and hypersensitivity, which are commonly seen in asthmatics [7, 8]. The goal of asthma treatment is to control asthma symptoms and maintain a normal level of activity while minimizing the risk of acute exacerbation and lung function damage [9]. A previous review of randomized controlled trials (RCTs) reported that AAHM plus routine pharmacotherapy (RP) compared to RP alone improved forced expiratory volume in 1 second $\left(\mathrm{FEV}_{1}\right)$ and asthmatic symptoms in patients with asthma [10]. However, there is a lack of evidence for direct comparison with placebo and no assessment of the quality of the evidence. In addition, important outcomes such as asthma control and acute exacerbation frequency were not evaluated in the previous systematic review.

Recently, the number and quality of randomized doubleblind placebo-controlled trials of AAHM for asthma have increased. Therefore, we systematically evaluated the evidence of multiple outcomes in order to determine whether adding AAHM was beneficial for patients with asthma.

\section{Methods}

2.1. Search Strategy. The following English and Chinese databases were searched from their inceptions until December 2020: PubMed, Allied and Complementary Medicine Database (AMED), Cumulative Index of Nursing and Allied Health Literature (CINAHL), Excerpta Medica Database (Embase), Cochrane Central Register of Controlled Trials (CENTRAL), Chongqing VIP (CQVIP), Chinese Biomedical Database (CBM), Wanfang Medicine Online (WFMO), and China National Knowledge Infrastructure (CNKI). The date and language of publication were not restricted. We also manually searched existing meta-analyses and scanned cited references in published studies to identify potentially relevant trials.
This strategy was intended to maximize the capture of citations for peer-reviewed publications relevant to three groups of search terms: condition (asthma and its synonyms); intervention (acupoint application and its synonyms), and study type (RCTs and its synonyms).

2.2. Inclusion Criteria. Studies were eligible if they satisfied the following criteria: (1) they were randomized doubleblind placebo-controlled trials; (2) patients had been diagnosed with asthma according to clinical symptoms and pulmonary function tests (e.g., Global Initiative for Asthma [11] or the Chinese Medical Association's diagnosis and treatment guidelines [12]; (3) they compared RP plus AAHM versus RP plus placebo or AAHM versus placebo; (4) the medicine used in AAHM was Chinese herbal medicine. RP included corticosteroids, bronchodilators, theophylline, and leukotriene receptor antagonists; and (5) trials reported at least one of the following outcome measures: lung function including $\mathrm{FEV}_{1}$, forced vital capacity (FVC), asthma control test (ACT), asthma quality of life questionnaire (AQLQ), total immunoglobulin $\mathrm{E}$ ( $\mathrm{IgE}$ ), eosinophil (EOS), and/or frequency of acute exacerbations.

2.3. Exclusion Criteria. Studies were excluded if they included: (1) patients with chronic obstructive pulmonary disease, interstitial lung disease, or other respiratory diseases; (2) AAHM had been combined with either acupuncture, acupoint injection, oral Chinese herbal medicine, or other therapies; and (3) Trials had used antibiotics, oral, or intravenous steroids as RP treatment.

2.4. Data Extraction. Two independent reviewers (YXC and YCZ) screened the studies according to the eligibility criteria, and disagreements were resolved by a third researcher (YBC). Titles and abstracts were first screened, and unrelated articles were excluded. Then, full articles were identified based on predefined inclusion and exclusion criteria.

After screening the studies, data were evaluated and extracted by two researchers (ZHW and YCZ) using a standard form. The form included the following items: (1) general details (e.g., title, first author, publication date, diagnostic criteria, and duration of asthma); (2) participants (e.g., sample size, age, sex, and severity of asthma); (3) methodological evaluation; (4) experimental and control interventions; (5) outcome measures; (6) dropouts and treatment/follow-up duration; and (7) adverse events. Attempts were made to contact corresponding authors via e-mail, mail, or phone when data were missing or incomplete.

2.5. Risk of Bias Assessment. The methodological quality of the included studies was assessed independently by two reviewers (ZHW and YJX) using the Cochrane Collaboration's risk of bias assessment tool [13]. The risk of bias in seven domains was assessed: (1) random sequence generation; (2) allocation concealment; (3) blinding of participants and personnel; (4) blinding of outcome assessors; (5) 
incomplete outcome data; (6) selective outcome reporting; and (7) other bias such as baseline balance, funding source, and conflicts of interest. Each risk of bias domain was assessed as low risk, high risk, or unclear risk. Any discrepancies were resolved by discussion with a third reviewer (LW or LL).

2.6. Statistical Analysis. All statistical analyses were conducted using Review Manager (RevMan 5.3.3) from the Cochrane Collaboration. Continuous data were calculated as mean difference (MD), with 95\% confidence intervals (CI). Visual inspection of forest plots, $p$-values, and $I^{2}$ statistics were used to evaluate statistical heterogeneity. An $I^{2}$ statistic over $50 \%\left(I^{2}>50 \%\right)$ was considered to be an indicator of high heterogeneity. If heterogeneity was low, a fixed-effects model was applied to estimate the treatment effect. If heterogeneity was high, a random-effects model was used. In addition, subgroup analysis and sensitivity analysis were used to explore potential heterogeneity. Funnel plot analysis was carried out to assess potential publication bias if there were ten or more studies in the pool.

2.7. Assessment of the Quality of Evidence. The Grading of Recommendations Assessment, Development, and Evaluation (GRADE) was used for developing and presenting summaries of evidence, and the quality of evidence in the meta-analysis was summarized and graded [14]. Although the quality of evidence is continuous, the GRADE method finally divided the quality of evidence group into four categories: high, moderate, low, and very low according to the level of evidence.

\section{Results}

3.1. Study Selection. A total of 5,723 studies were initially retrieved from English and Chinese databases. After 3,034 duplicates were removed, the remaining 2,689 were screened. The full texts of 110 were retrieved after title and abstract screening. Full-text evaluation removed another 93 unrelated studies. A total of 16 eligible RCTs [15-30] (1,730 participants) were reviewed and included in the metaanalysis (Figure 1).

3.2. Study Characteristics. All studies [15-30] were conducted in China and retrieved from Chinese databases. In four studies $[16-18,20]$, the participants were children; 12 studies [15, 19, 21-30] included adults. Participants were diagnosed with asthma according to the Chinese Medical Association's diagnosis and treatment guidelines in all studies.

Ten studies [15, 18-26] applied the AAHM treatment during the hottest days of summer (San-Fu days also called dog days, between mid-July and mid-August); two studies $[16,17]$ were conducted during the hottest days of summer and the coldest days of winter (San-Jiu days, between early-January and mid-January); and four studies [27-30] did not mention the time of year of

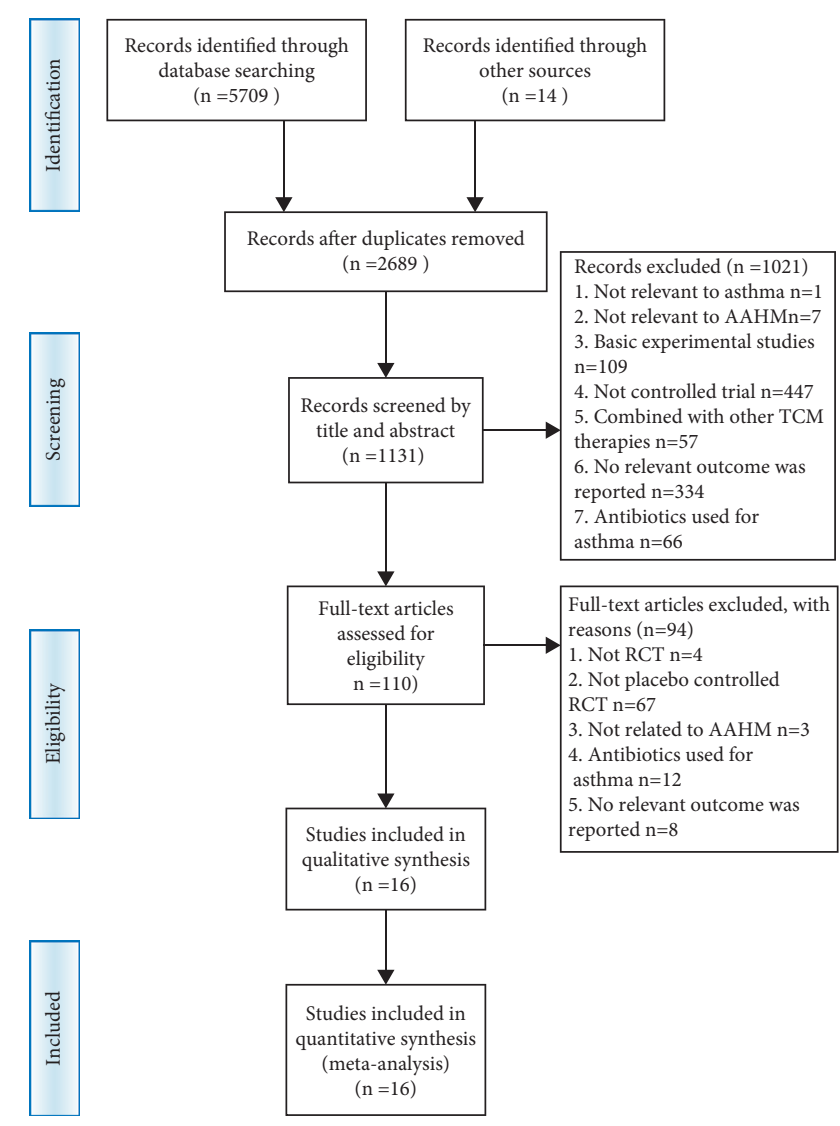

Figure 1: Flow diagram of the study selection process.

treatment. Herbal medicines included Xi Xin (Herbal Asari), Bai Jie Zi (Semen Brassicae), Gan Sui (Radix Kansui), Yan Hu Suo (Rhizoma Corydalis), and so on. Feishu (BL13), Dingchuan (EX-B1), Tiantu (CV22), Shenshu (BL23), and Dazhui (GV14) were commonly used as acupoints across included studies. RP included corticosteroids, bronchodilators, leukotriene receptor antagonist, and so on.

Participants enrolled in the studies were randomly divided into two groups: the treatment groups included AAHM alone or AAHM plus RP, and control groups included placebo AAHM alone or placebo plus RP. Six studies $[15-17,19,25,30]$ compared AAHM plus RP with placebo plus RP, and ten studies [18, 20-24, 26-29] compared AAHM alone with placebo alone. Treatment duration ranged from 4 weeks to 4 years. Studies with a treatment duration of 1 year or more were defined as long-term efficacy observation $[15,16,19,20,22,23,25,26]$, and those with a duration of less than 1 year were short-term efficacy observation $[17,18,21,24,27-30]$. Twelve studies [15-20, 23, 25, 27-30] reported lung function parameters (including at least one of $\mathrm{FEV}_{1}$ and $\mathrm{FEV}_{1} / \mathrm{FVC}$ ); three studies [16, 19, 24] evaluated total IgE; four studies $[15,19,21,26]$ reported acute exacerbations; two studies $[16,20,29]$ reported EOS; and three studies [25, 27, 28] assessed AQLQ; and two studies [15, 23] assessed ACT. Characteristics of all included studies are summarized in Tables 1 and 2 . 
TABLE 1: Characteristics of included studies.

\begin{tabular}{|c|c|c|c|c|}
\hline First author, year [ref.] & Country & Age (years, mean $\pm \mathrm{SD}$ ) & Sample size $(\mathrm{R} / \mathrm{A})$ & Asthma history Mean \pm SD (years) \\
\hline \multirow[t]{2}{*}{ Yang, 2018 [15] } & China & $\mathrm{T}: 56.67 \pm 10.62$ & T: $30 / 30$ & $\mathrm{~T}: 10.17 \pm 6.79$ \\
\hline & & C: $56.80 \pm 12.32$ & C: $30 / 30$ & $C: 9.87 \pm 5.29$ \\
\hline \multirow{2}{*}{ Wang, 2017 [16] } & China & $\mathrm{T}: 8.0 \pm 2.8$ & $\mathrm{~T}: 60 / 60$ & $\mathrm{~T}: 1.68 \pm 0.88$ \\
\hline & & C: $7.5 \pm 2.6$ & C: $60 / 60$ & $C: 1.67 \pm 0.67$ \\
\hline \multirow[t]{2}{*}{ Wang (b), 2017 [17] } & China & $\mathrm{T}: 7.6 \pm 2.8$ & $\mathrm{~T}: 50 / 50$ & $\mathrm{~T}: 1.58 \pm 0.78$ \\
\hline & & $C: 7.5 \pm 2.6$ & C: $50 / 50$ & $C: 1.57 \pm 0.72$ \\
\hline \multirow[t]{2}{*}{ Ma, 2016 [18] } & China & $\mathrm{T}: 7.6 \pm 2.5$ & $\mathrm{~T}: 54 / 54$ & $\mathrm{~T}: 2.9 \pm 1.4$ \\
\hline & & C: $8.1 \pm 2.7$ & C: $53 / 53$ & $\mathrm{C}: 3.2 \pm 1.5$ \\
\hline \multirow[t]{2}{*}{ Wang, 2016 [19] } & China & $\mathrm{T}: 48 \pm 6.4$ & $\mathrm{~T}: 44 / 44$ & $\mathrm{~T}: 9.5 \pm 3.5$ \\
\hline & & C: $47 \pm 6.9$ & C: $44 / 44$ & C: $7.3 \pm 3.1$ \\
\hline \multirow[t]{2}{*}{ Shi, 2014 [20] } & China & $\mathrm{T}: 4.8 \pm 0.3$ & $\mathrm{~T}: 46 / 46$ & $\mathrm{~T}: 7.50 \pm 3.30$ \\
\hline & & C: $4.9 \pm 0.5$ & C: $46 / 46$ & $\mathrm{C}: 7.30 \pm 3.10$ \\
\hline \multirow[t]{2}{*}{ Chang, 2014 [21] } & China & $\mathrm{T}: 37.21 \pm 12.90$ & $\mathrm{~T}: 75 / 75$ & $\mathrm{~T}: 18.28 \pm 8.90 \mathrm{M}$ \\
\hline & & C: $38.91 \pm 11.85$ & C: $68 / 68$ & $\mathrm{C}: 19.91 \pm 9.18 \mathrm{M}$ \\
\hline \multirow[t]{2}{*}{ Liu, 2018 [22] } & China & $\mathrm{T}: 50 \pm 15$ & $\mathrm{~T}: 30 / 30$ & $\mathrm{~T}: 8.7 \pm 4.7$ \\
\hline & & C: $49 \pm 12$ & C: $30 / 30$ & $\mathrm{C}: 9.1 \pm 4.7$ \\
\hline \multirow[t]{2}{*}{ Tian, 2020 [23] } & China & $\mathrm{T}: 54.82 \pm 11.77$ & T: $56 / 56$ & $\mathrm{~T}: 2-7$ \\
\hline & & C: $53.6 \pm 13.28$ & C: $55 / 55$ & C: $3-7$ \\
\hline \multirow[t]{2}{*}{ Shang, 2013 [24] } & China & $\mathrm{T}: 35.0 \pm 11.0$ & $\mathrm{~T}: 80 / 80$ & T: NR \\
\hline & & $\mathrm{C}: 32.0 \pm 12.0$ & C: $40 / 40$ & C: NR \\
\hline \multirow[t]{2}{*}{$\mathrm{Lu}, 2011[25]$} & China & $\mathrm{T}: 46.82 \pm 10.11$ & $\mathrm{~T}: 35 / 35$ & $\mathrm{~T}: 4.76 \pm 1.23$ \\
\hline & & C: $47.42 \pm 11.84$ & C: $35 / 35$ & $\mathrm{C}: 5.42 \pm 1.74$ \\
\hline \multirow[t]{2}{*}{ Bai, $2010[26]$} & China & $\mathrm{T}: 19-52$ & $\mathrm{~T}: 42 / 42$ & $\mathrm{~T}: 0.25-6$ \\
\hline & & C: $25-51$ & C: $42 / 42$ & C: $0.16-5$ \\
\hline \multirow{2}{*}{ Yao, 2009 [27] } & China & $\mathrm{T}: 45.3 \pm 12.0$ & $\mathrm{~T}: 125 / 125$ & $\mathrm{~T}: 12.4 \pm 3.4$ \\
\hline & & C: $45.4 \pm 12.7$ & C: $63 / 63$ & C: $11.8 \pm 4.7$ \\
\hline \multirow[t]{2}{*}{$\mathrm{Yu}, 2009$ [28] } & China & NR & T: $33 / 33$ & NR \\
\hline & & & C: $17 / 17$ & \\
\hline \multirow{2}{*}{ Yao, 2007 [29] } & China & $\mathrm{T}: 46.2 \pm 12.1$ & $\mathrm{~T}: 44 / 44$ & NR \\
\hline & & $C: 51.0 \pm 11.8$ & C: $22 / 22$ & \\
\hline \multirow[t]{2}{*}{ Mi, $2005[30]$} & China & $\mathrm{T}: 18-65$ & $\mathrm{~T}: 181 / 181$ & $\mathrm{~T}: 0.16-20$ \\
\hline & & C: $18-65$ & C: $181 / 181$ & C: $0.08-30$ \\
\hline
\end{tabular}

Abbreviations: T: treatment, C: control, R: number of subjects randomized, A: number of subjects analyzed, M: month, NR: not reported, and SD: standard deviation.

3.3. Risk of Bias. Nine studies [15, 20-23, 25, 27-29] explicitly described methods for random sequence generation and were judged as having a low risk of selection bias. Two studies [23, 27] mentioned the methods of allocation concealment were judged to be at unclear risk. Sixteen studies [15-30] were judged at low risk of bias in the blinding of participants and personnel because acupoint application of placebo was reported. Incomplete outcome data were assessed at low risk if there was no missing data or dropouts were balanced between groups. Any studies without incomplete outcome data were judged to be at low risk. All studies were judged at low risk of bias for selective outcome reporting. Other biases including baseline imbalance, research funding, and conflicts of interest were at low risk in ten studies $[16,17,21,22,24-26,28-30]$. The risk of bias is summarized in Figure S1.

3.4. Publication Bias. The tests for funnel plot asymmetry should not be used when there are fewer than ten studies in the meta-analysis. Therefore, we did not assess potential publication bias.

\subsection{Results of AAHM plus RP versus Placebo plus $R P$}

3.5.1. Lung Function. Spirometry is used to demonstrate airway obstruction to confirm diagnosis as well as monitor asthma over time. Lung function $\mathrm{FEV}_{1}$ and FVC are the primary measures used to assess asthma. Increases of more than 0.23 litres or $10 \%$ indicate clinically important improvement $[31,32]$. Improving lung function is one of the primary objectives of asthma management. To objectively measure asthma over time, regular monitoring of spirometry is commonly used in the clinical and research setting.

$\mathrm{FEV}_{1}(\%)$ was assessed in six studies (549 participants) that were judged as low-quality evidence (Tables 3 and 4). The forest plot of long-term efficacy showed $\mathrm{FEV}_{1}$ (\%) increased, although the heterogeneity was high (MD $11.80 \%$, $95 \%$ CI 2.84 to $20.76, I^{2}=99 \%$ ), and the forest plot of shortterm efficacy had a similar result (MD 10.57\%, 95\% CI 8.40 to 12.74; Figure 2). Low-quality evidence from one study evaluated the short-term efficacy of $\mathrm{FEV}_{1}$ (L; Table 4), and the result indicated that AAHM plus RP was better than placebo plus RP (MD 0.80 L, 95\% CI 0.55 to 1.05; Figure S2). 
TABLE 2: Interventions and outcome assessments.

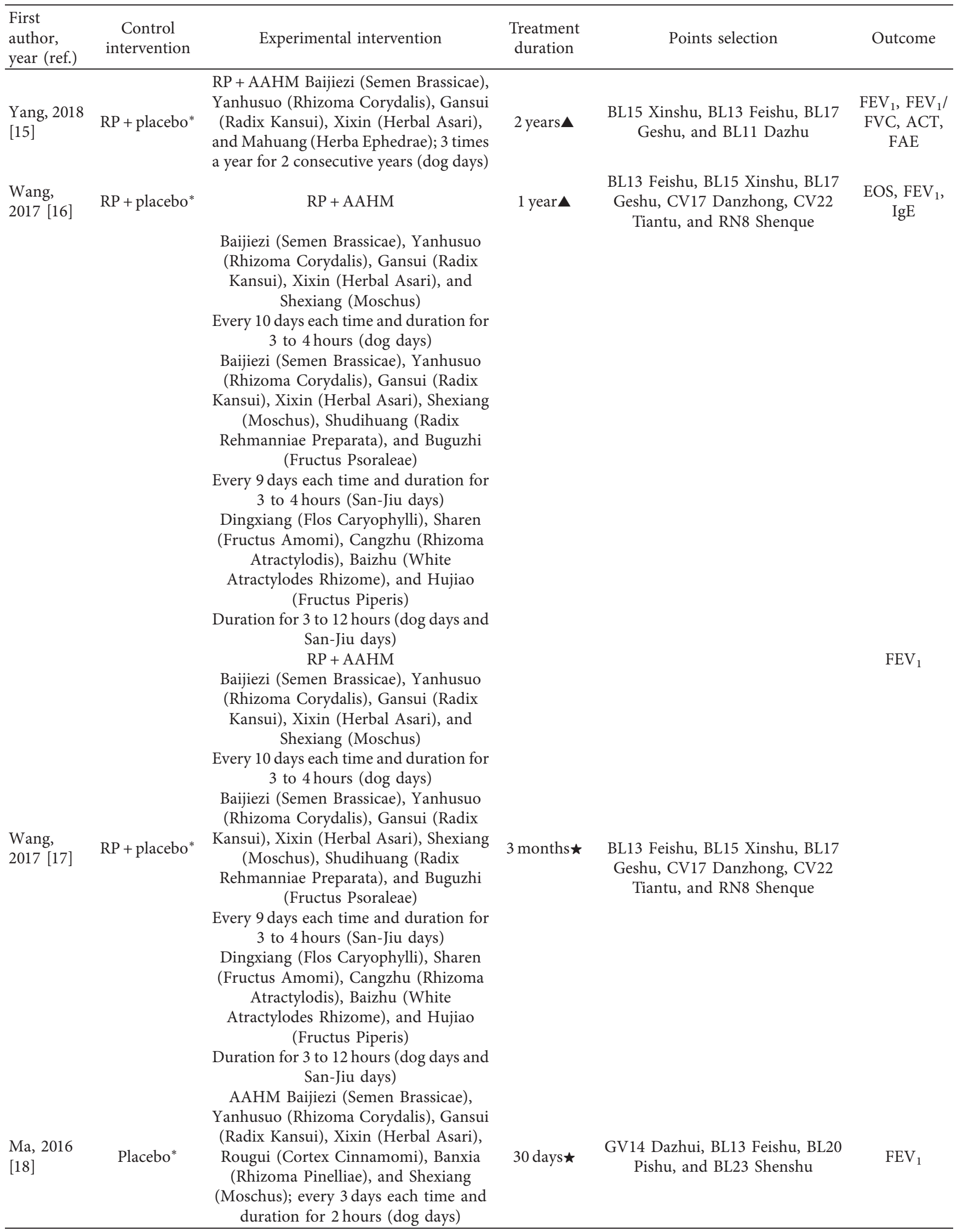


TABle 2: Continued.

\begin{tabular}{|c|c|c|c|c|c|}
\hline $\begin{array}{l}\text { First } \\
\text { author, } \\
\text { year (ref.) }\end{array}$ & $\begin{array}{l}\text { Control } \\
\text { intervention }\end{array}$ & Experimental intervention & $\begin{array}{l}\text { Treatment } \\
\text { duration }\end{array}$ & Points selection & Outcome \\
\hline $\begin{array}{l}\text { Wang, } \\
2016[19]\end{array}$ & $\mathrm{RP}+$ placebo* $^{*}$ & $\begin{array}{c}\text { RP + AAHM Baijiezi (Semen Brassicae), } \\
\text { Yanhusuo (Rhizoma Corydalis), Xixin } \\
\text { (Herbal Asari), Gansui (Radix Kansui), } \\
\text { and Shexiang (Moschus); every } 10 \text { days } \\
\text { each time and duration for } 2 \text { to } 6 \text { hours } \\
\text { (dog days) }\end{array}$ & 1 year $\boldsymbol{\Delta}$ & $\begin{array}{c}\text { First dog days: } \\
\text { BL12 Fengmen and BL13 Feishu } \\
\text { EX-B1 Dingchuan } \\
\text { Middle dog days: } \\
\text { BL20 Pishu and BL14 Jueyinshu } \\
\text { GV14 Dazhui } \\
\text { End dog days: } \\
\text { BL23 Shenshu and BL11 Dazhu } \\
\text { BL43 Gaohuang }\end{array}$ & $\mathrm{FEV}_{1}, \mathrm{IgE}$ \\
\hline $\begin{array}{l}\text { Shi, } 2014 \\
{[20]}\end{array}$ & Placebo* & $\begin{array}{l}\text { AAHM Baijiezi (Semen Brassicae), } \\
\text { Yanhusuo (Rhizoma Corydalis), Gansui } \\
\text { (Radix Kansui), Xixin (Herbal Asari), } \\
\text { and Fangfeng (Radix Saposhnikoviae); } \\
\text { every } 10 \text { days each time and duration for } \\
10 \text { minutes (dog days) }\end{array}$ & 1 year $\mathbf{\Delta}$ & $\begin{array}{l}\text { BL15 Xinshu, BL13 Feishu, and } \\
\text { BL17 Geshu }\end{array}$ & $\begin{array}{l}\mathrm{EOS} \mathrm{FEV}_{1}, \\
\mathrm{FEV}_{1} / \mathrm{FVC}\end{array}$ \\
\hline \multirow[t]{2}{*}{$\begin{array}{l}\text { Chang, } \\
2014[21]\end{array}$} & Placebo* & 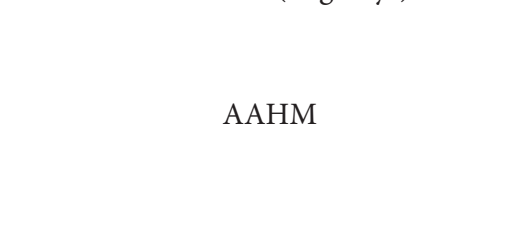 & 32 days $\star$ & $\begin{array}{c}\text { Scheme 1: GV14 Dazhui; BL13 } \\
\text { Feishu; EX-B1 Dingchuan; CV22 } \\
\text { Tiantu; BL12 Fengmen; Scheme 2: } \\
\text { BL13 Feishu; BL43 Gaohuang; BL20 } \\
\text { Pishu; BL23 Shenshu; CV17 } \\
\text { Danzhong. }\end{array}$ & FAE \\
\hline & & $\begin{array}{c}\text { Scheme 1: } \\
\text { Baijiezi (Semen Brassicae), Yanhusuo } \\
\text { (Rhizoma Corydalis), Gansui (Radix } \\
\text { Kansui), Xixin (Herbal Asari), Fangfeng } \\
\text { (Radix Saposhnikoviae), Wuweizi } \\
\text { (Fructus Schisandrae Chinensis), and } \\
\text { Bingpian (Borneclum Syntheticum) } \\
\text { Scheme 2: } \\
\text { Baijiezi (Semen Brassicae), Yanhusuo } \\
\text { (Rhizoma Corydalis), Gansui (Radix } \\
\text { Kansui), Xixin (Herbal Asari), Ganjiang } \\
\text { (Dried Ginger), Rougui (Cortex } \\
\text { Cinnamomi), Yinyanghuo (Herba } \\
\text { Epimedii), and Bingpian (Borneclum } \\
\text { Syntheticum) } \\
\text { (Schemes } 1 \text { and } 2 \text { were used alternately) } \\
\text { Every } 4 \text { days each time and duration for } \\
3 \text { to } 5 \text { hours (dog days) }\end{array}$ & & & \\
\hline $\begin{array}{l}\text { Liu, } 2018 \\
{[22]}\end{array}$ & Placebo* & $\begin{array}{l}\text { AAHM Baijiezi (Semen Brassicae), } \\
\text { Yanhusuo (Rhizoma Corydalis), Gansui } \\
\text { (Radix Kansui), and Xixin (Herbal } \\
\text { Asari); every } 10 \text { days each time and } \\
\text { duration for } 4 \text { hours (dog days) }\end{array}$ & 1 year $\mathbf{\Delta}$ & $\begin{array}{l}\text { BL15 Xinshu, BL13 Feishu, and } \\
\text { BL17 Geshu }\end{array}$ & FAE \\
\hline $\begin{array}{l}\text { Tian, } 2020 \\
\text { [23] }\end{array}$ & $\mathrm{RP}+$ placebo* & $\begin{array}{c}\text { RP + AAHM Baijiezi (Semen Brassicae), } \\
\text { Yanhusuo (Rhizoma Corydalis), Gansui } \\
\text { (Radix Kansui), and Xixin (Herbal } \\
\text { Asari); } 3 \text { times a year for } 2 \text { consecutive } \\
\text { years and duration for } 2 \text { to } 4 \text { hours (dog } \\
\text { days) }\end{array}$ & 2 years & $\begin{array}{l}\text { BL15 Xinshu, BL13 Feishu, BL17 } \\
\text { Geshu, BL20 Pishu, ST40 Fenglong }\end{array}$ & FAE, FEV $_{1}$ \\
\hline $\begin{array}{l}\text { Shang, } \\
2013[24]\end{array}$ & Placebo* & $\begin{array}{l}\text { AAHM Xixin (Herbal Asari), Baijiezi } \\
\text { (Semen Brassicae), Gansui (Radix } \\
\text { Kansui), and Mahuang (Herba } \\
\text { Ephedrae); twice a week and duration } \\
\text { for } 1 \text { hour (dog days) }\end{array}$ & 4 weeks $\star$ & $\begin{array}{l}\text { Along governor vessel and bladder } \\
\text { meridian of foot taiyang }\end{array}$ & $\operatorname{IgE}$ \\
\hline
\end{tabular}


TABLE 2: Continued.

\begin{tabular}{|c|c|c|c|c|c|}
\hline $\begin{array}{l}\text { First } \\
\text { author, } \\
\text { year (ref.) }\end{array}$ & $\begin{array}{c}\text { Control } \\
\text { intervention }\end{array}$ & Experimental intervention & $\begin{array}{l}\text { Treatment } \\
\text { duration }\end{array}$ & Points selection & Outcome \\
\hline $\begin{array}{l}\mathrm{Lu}, 2011 \\
{[25]}\end{array}$ & $\mathrm{RP}+$ placebo* & $\begin{array}{c}\text { RP + AAHM Baijiezi (Semen Brassicae), } \\
\text { Yanhusuo (Rhizoma Corydalis), Gansui } \\
\text { (Radix Kansui), and Xixin (Herbal } \\
\text { Asari); every } 10 \text { days each time (dog } \\
\text { days) }\end{array}$ & 3 years $\Delta$ & $\begin{array}{c}\text { Scheme 1: } \\
\text { CV22 Tiantu and GV14 Dazhui } \\
\text { BL13 Feishu and BL20 Pishu } \\
\text { RN8 Shenshu and ST36 Zusanli } \\
\text { Scheme 2: } \\
\text { CV17 Danzhong and BL42 Pohu } \\
\text { ST40 Fenglong and BL49 Yishe } \\
\text { EX-B1 Dingchuan and BL52 Zhishi } \\
\text { (Schemes } 1 \text { and 2 were used } \\
\text { alternately) }\end{array}$ & $\begin{array}{c}\mathrm{FEV}_{1}, \\
\mathrm{AQLQ} \\
\mathrm{FEV}_{1} / \mathrm{FVC}\end{array}$ \\
\hline $\begin{array}{l}\text { Bai, } 2010 \\
{[26]}\end{array}$ & Placebo* & $\begin{array}{l}\text { AAHM Baijiezi (Semen Brassicae), } \\
\text { Gansui (Radix Kansui), Fangfeng (Radix } \\
\text { Saposhnikoviae), Huangqin (Radix } \\
\text { Scutellariae), and Dilong (Pheretima); } \\
\text { every } 2 \text { or } 3 \text { days each time (dog days) }\end{array}$ & 3 years $\Delta$ & $\begin{array}{l}\text { BL23 Shenshu, BL13 Feishu, EX-B1 } \\
\text { Dingchuan, BL43 Gaohuang }\end{array}$ & FAE \\
\hline $\begin{array}{l}\text { Yao, } 2009 \\
{[27]}\end{array}$ & Placebo* & $\begin{array}{l}\text { AAHM Baijiezi (Semen Brassicae), } \\
\text { Hujiao (Fructus Piperis), Xixin (Herbal } \\
\text { Asari), and Baizhi (Radix Angelicae } \\
\text { Dahuricae); every } 3 \text { or } 4 \text { days each time } \\
\text { and duration for } 4 \text { to } 4 \text { hours ( } 4 \text { weeks) }\end{array}$ & 4 weeks $\star$ & $\begin{array}{c}\text { Scheme 1: } \\
\text { CV22 Tiantu and GV14 Dazhui; } \\
\text { BL13 Feishu and BL20 Pishu } \\
\text { Scheme 2: } \\
\text { CV22 Tiantu and CV17 Danzhong } \\
\text { ST36 Zusanli and RN6 Qihai } \\
\text { RN4 Guanyuan } \\
\text { (Schemes land 2 were used } \\
\text { alternately) }\end{array}$ & $\begin{array}{c}\text { AQLQ, } \\
\mathrm{FEV}_{1} / \mathrm{FVC}\end{array}$ \\
\hline $\begin{array}{l}\text { Yu, } 2009 \\
{[28]}\end{array}$ & Placebo* & $\begin{array}{l}\text { AAHM Baijiezi (Semen Brassicae), } \\
\text { Hujiao (Fructus Piperis), Xixin (Herbal } \\
\text { Asari), Banfenghe (Pterospermum } \\
\text { heterophyllum Hance), and Baizhi } \\
\text { (Radix Angelicae Dahuricae); twice a } \\
\text { week and duration for } 4 \text { hours (4 weeks) }\end{array}$ & 4 weeks $\star$ & $\begin{array}{c}\text { Scheme 1: } \\
\text { CV22 Tiantu and GV14 Dazhui } \\
\text { BL13 Feishu and BL20 Pishu } \\
\text { Scheme 2: } \\
\text { CV22 Tiantu and CV17 Danzhong } \\
\text { ST36 Zusanli and RN6 Qihai } \\
\text { RN4 Guanyuan } \\
\text { (Schemes } 1 \text { and 2 were used } \\
\text { alternately) } \\
\text { Scheme 1: }\end{array}$ & $\begin{array}{l}\mathrm{FEV}_{1}, \mathrm{FEV}_{1} / \\
\mathrm{FVC}, \mathrm{AQLQ},\end{array}$ \\
\hline $\begin{array}{l}\text { Yao, } 2007 \\
{[29]}\end{array}$ & Placebo* & $\begin{array}{l}\text { AAHM Baijiezi (Semen Brassicae), } \\
\text { Hujiao (Fructus Piperis), Xixin (Herbal } \\
\text { Asari), Banfenghe (Pterospermum } \\
\text { heterophyllum Hance), and Baizhi } \\
\text { (Radix Angelicae Dahuricae); twice a } \\
\text { week and duration for } 4 \text { hours (4 weeks) }\end{array}$ & 4 weeks $\star$ & $\begin{array}{l}\text { CV22 Tiantu and GV14 Dazhui } \\
\text { BL13 Feishu and BL20 Pishu } \\
\text { Scheme 2: } \\
\text { CV22 Tiantu and CV17 Danzhong } \\
\text { ST36 Zusanli and RN6 Qihai } \\
\text { RN4 Guanyuan } \\
\text { (Schemes } 1 \text { and 2 were used } \\
\text { alternately) }\end{array}$ & EOS, $\mathrm{FEV}_{1}$ \\
\hline $\begin{array}{l}\mathrm{Mi}, 2005 \\
{[30]}\end{array}$ & $\mathrm{RP}+$ placebo* & $\begin{array}{l}\text { RP + AAHM, Baijiezi (Semen Brassicae), } \\
\text { Yanhusuo (Rhizoma Corydalis), Gansui } \\
\text { (Radix Kansui), and Xixin (Herbal } \\
\text { Asari); every } 10 \text { days each time and } \\
\text { duration for } 1 \text { hour ( } 3 \text { months) }\end{array}$ & 3 months $\star$ & $\begin{array}{c}\text { Scheme 1: } \\
\text { BL13 Feishu and BL21 Weishu } \\
\text { CV17 Danzhong and BL52 Zhishi } \\
\text { Scheme 2: } \\
\text { BL20 Pishu and BL43 Gaohuang } \\
\text { BL12 Fengmen and CV22 Tiantu } \\
\text { Scheme3: } \\
\text { BL23 Shenshu and RN12 Zhongwan } \\
\text { EX-B1 Dingchuan and BL23 Xinshu } \\
\text { (Schemes 1, 2, and } 3 \text { were used } \\
\text { alternately) }\end{array}$ & $\mathrm{FEV}_{1} / \mathrm{FVC}$ \\
\hline
\end{tabular}

Abbreviations: ACT: asthma control test, AAHM: acupoint application of herbal medicine, AQLQ: asthma quality of life questionnaire, FAE: frequency of acute exacerbations, IgE: Immunoglobulin E, RP: routine pharmacotherapy, EOS: eosinophil, $\mathrm{FEV}_{1}$ : forced expiratory volume in 1 second, and FVC: forced vital capacity. ${ }^{*}$ The application method and point selection are the same as the experimental intervention. $\mathbf{\Delta}$ Long-term efficacy observation. $\star$ Short-term efficacy observation. 
TABLE 3: Summary of GRADE evidence: Comparison of long-term efficacy between AAHM plus RP and placebo plus RP.

\begin{tabular}{|c|c|c|c|c|}
\hline \multirow[b]{2}{*}{ Outcomes } & \multirow{2}{*}{$\begin{array}{c}\text { Number of participants (number of } \\
\text { Studies) }\end{array}$} & \multirow{2}{*}{$\begin{array}{l}\text { Quality of evidence } \\
\text { (GRADE) }\end{array}$} & \multicolumn{2}{|r|}{ Absolute effect } \\
\hline & & & $\begin{array}{l}\text { Control group } \\
(\text { mean })\end{array}$ & $\begin{array}{c}\text { Comparison between groups ( } 95 \% \\
\text { CI ) }\end{array}$ \\
\hline $\mathrm{FEV}_{1}(\%)$ & 449 (5 RCTs) & $\oplus \oplus \bigcirc \bigcirc$ Low 1,4 & $70.70 \%$ & $\uparrow 11.80(2.84,20.76)$ \\
\hline $\begin{array}{l}\mathrm{FEV}_{1} / \\
\mathrm{FVC}\end{array}$ & 130 (2 RCTs) & $\oplus \bigcirc \bigcirc \bigcirc$ Very low $1,2,3$ & $67.47 \%$ & No statistical significance \\
\hline FAE & 148 (2 RCTs) & $\oplus \bigcirc \bigcirc \bigcirc$ Very Low $2,3,4$ & 3.03 times & No statistical signific \\
\hline $\mathrm{ACT}$ & 171 (2 RCTs) & $\oplus \bigcirc \bigcirc \bigcirc$ Very Low $1,2,3$ & 20.71 points & No statistical significance \\
\hline AQLQ & $70(1 \mathrm{RCT})$ & $\oplus \oplus \oplus \bigcirc$ Moderate 3 & 109.61 points & $\uparrow 6.53(2.70,10.36)$ \\
\hline Total IgE & 208 (2 RCTs) & $\oplus \bigcirc \bigcirc \bigcirc$ Very Low $1,2,3,4$ & $693.62 \mathrm{IU} / \mathrm{ml}$ & No statistical significance \\
\hline EOS & $120(1 \mathrm{RCT})$ & $\oplus \oplus \bigcirc \bigcirc$ Low 3,4 & $0.61 \times 0.09 / \mathrm{L}$ & $\downarrow-0.31(-0.37,-0.25)$ \\
\hline
\end{tabular}

Abbreviations: ACT: asthma control test, AQLQ: asthma quality of life questionnaire, FAE: frequency of acute exacerbations, IgE: immunoglobulin E, EOS: eosinophil, $\mathrm{FEV}_{1}$ : Forced expiratory volume in 1 second, and FVC: forced vital capacity. 1 . Considerable statistical heterogeneity, 2. the credible interval contains invalid values and wide interval limits the accuracy of the results, 3 . insufficient sample size limits the accuracy of the results, and 4 . unclear sequence generation.

TABLE 4: Summary of GRADE evidence: comparison of short-term efficacy between AAHM plus RP and placebo plus RP

\begin{tabular}{|c|c|c|c|c|}
\hline \multirow[b]{2}{*}{ Outcomes } & \multirow[b]{2}{*}{$\begin{array}{c}\text { Number of participants (number of } \\
\text { studies) }\end{array}$} & \multirow[b]{2}{*}{$\begin{array}{l}\text { Quality of evidence } \\
\text { (GRADE) }\end{array}$} & \multicolumn{2}{|r|}{ Absolute effect } \\
\hline & & & $\begin{array}{l}\text { Control group } \\
(\text { mean })\end{array}$ & $\begin{array}{c}\text { Comparison between groups ( } 95 \% \\
\text { CI ) }\end{array}$ \\
\hline $\mathrm{FEV}_{1}(\%)$ & $100(1 \mathrm{RCT})$ & $\oplus \oplus \bigcirc \bigcirc$ Low 3,4 & $78.61 \%$ & $\uparrow 10.57(8.40,12.74)$ \\
\hline $\mathrm{FEV}_{1}(\mathrm{~L})$ & $88(1 \mathrm{RCT})$ & $\oplus \oplus \bigcirc \bigcirc$ Low 3,4 & $2.01 \mathrm{~L}$ & $\uparrow 0.80(0.55,1.05)$ \\
\hline $\begin{array}{l}\mathrm{FEV}_{1} / \\
\mathrm{FVC}\end{array}$ & $362(1 \mathrm{RCT})$ & $\oplus \oplus \bigcirc \bigcirc$ Low 3,4 & $72.11 \%$ & $\uparrow 7.77(6.07,9.47)$ \\
\hline
\end{tabular}

Abbreviations: ACT: asthma control test, AQLQ: asthma quality of life questionnaire, FAE: frequency of acute exacerbations, IgE: immunoglobulin E, EOS: eosinophil, $\mathrm{FEV}_{1}$ : forced expiratory volume in 1 second, and FVC: forced vital capacity. 1. Considerable statistical heterogeneity, 2. the credible interval contains invalid values and wide interval limits the accuracy of the results, 3 . insufficient sample size limits the accuracy of the results, and 4 . unclear sequence generation.

\begin{tabular}{|c|c|c|c|c|c|c|c|c|c|c|}
\hline \multirow[b]{2}{*}{ Study or Subgroup } & \multicolumn{3}{|c|}{ Treatment group } & \multicolumn{3}{|c|}{ Control group } & \multirow[b]{2}{*}{ Weight } & \multirow{2}{*}{$\begin{array}{l}\text { Mean Difference } \\
\text { IV, Random, 95\% CI }\end{array}$} & \multirow{2}{*}{\multicolumn{2}{|c|}{$\begin{array}{l}\text { Mean Difference } \\
\text { IV, Random, } 95 \% \text { CI }\end{array}$}} \\
\hline & Mean & SD & Total & Mean & SD & Total & & & & \\
\hline \multicolumn{11}{|c|}{ Long-term efficacy: $A A H M$ plus RP vs Palcebo plus RP } \\
\hline $\operatorname{Lu} 2011^{[25]}$ & 79.41 & 13.87 & 35 & 59.61 & 11.52 & 35 & $12.0 \%$ & $19.80[13.83,25.77]$ & & $\hookrightarrow-$ \\
\hline Tian $2020^{[23]}$ & 78.55 & 1.74 & 56 & 77.69 & 1.8 & 55 & $13.4 \%$ & $0.86[0.20,1.52]$ & & \\
\hline Wang $2016^{[25]}$ & 86.71 & 6.66 & 44 & 72.29 & 6.8 & 44 & $13.1 \%$ & $14.42[11.61,17.23]$ & & - \\
\hline Wang $2017^{[25]}$ & 89.08 & 5.67 & 60 & 71.51 & 5.19 & 60 & $13.2 \%$ & $17.57[15.63,19.51]$ & & $=$ \\
\hline $\begin{array}{l}\text { Yang } 20188^{[15]} \\
\text { Subtotal }(95 \% \text { CI) }\end{array}$ & 79.39 & 7.48 & $\begin{array}{l}30 \\
225\end{array}$ & 72.39 & 5.32 & $\begin{array}{c}30 \\
224\end{array}$ & $\begin{array}{l}13.0 \% \\
64.7 \%\end{array}$ & $\begin{array}{c}7.00[3.72,10.28] \\
11.80[2.84,20.76]\end{array}$ & & 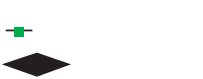 \\
\hline \multicolumn{11}{|c|}{$\begin{array}{l}\text { Heterogeneity: } \mathrm{Tau}^{2}=101.53 ; \mathrm{Chi}^{2}=353.55, \mathrm{df}=4(P<0.00001) ; I^{2}=99 \% \\
\text { Test for overall effect: } Z=2.58(P=0.010)\end{array}$} \\
\hline \multicolumn{11}{|c|}{$\begin{array}{l}\text { Heterogeneity: Not applicable } \\
\text { Test for overall effect: } Z=9.55(P<0.00001) \\
\text { Short-term efficacy: } A A H M \text { vs Palcebo }\end{array}$} \\
\hline Yao $2009^{[27]}$ & 79.17 & 20.66 & 125 & 68.38 & 13.14 & 63 & $12.4 \%$ & $10.79[5.93,15.65]$ & & - \\
\hline $\begin{array}{l}\text { Yu } 2009^{[28]} \\
\text { Subtotal }(95 \% \text { CI) }\end{array}$ & 81.02 & 21.98 & $\begin{array}{c}33 \\
158\end{array}$ & 88.72 & 16.31 & $\begin{array}{l}17 \\
80\end{array}$ & $\begin{array}{c}9.7 \% \\
22.1 \%\end{array}$ & $\begin{array}{l}-7.70[-18.49,3.09] \\
2.20[-15.88,20.27]\end{array}$ & & \\
\hline \multicolumn{11}{|c|}{$\begin{array}{l}\text { Heterogeneity: } \mathrm{Tau}^{2}=152.72 ; \mathrm{Ch}^{2}=9.38, \mathrm{df}=1(P=0.002) ; I^{2}=89 \% \\
\text { Test for overall effect: } Z=0.24(P=0.81)\end{array}$} \\
\hline \multicolumn{11}{|c|}{$\begin{array}{l}\text { Heterogeneity: } \mathrm{Tau}^{2}=79.05 ; \mathrm{Chi}^{2}=404.31, \mathrm{df}=7(P<0.00001) ; I^{2}=98 \% \\
\text { Test for overall effect: } Z=2.95(P=0.003) \\
\text { Test for subgroup differences: } C h i^{2}=0.90, \mathrm{df}=2(P=0.64) ; I^{2}=0 \%\end{array}$} \\
\hline & & & & & & & & & $-20-10$ & 1020 \\
\hline & & & & & & & & & Control group & Treatment group \\
\hline
\end{tabular}


$\mathrm{FEV}_{1} / \mathrm{FVC}$ was assessed in two studies (long-term efficacy) with very low-quality evidence (Table 3 ), and the forest plot showed no significant difference between the groups, and the heterogeneity was high (MD 9.77, 95\% CI -4.75 to 24.28, $I^{2}=96 \%$; Figure S3). However, another short-term efficacy observation with low-quality evidence showed that the treatment group was better than the control group in terms of $\mathrm{FEV}_{1} / \mathrm{FVC}$ (MD 7.77, 95\% CI 6.07 to 9.47; Table 4 and Figure S3).

3.5.2. Frequency of Acute Exacerbations. Exacerbations are characterized by acute worsening of wheezing, coughing, shortness of breath, chest tightness, and lung function reduction. Exacerbations put patients at risk of respiratory distress, hypoxemia, and airflow obstruction and are the most important marker of asthma management and treatment effectiveness [33]. Reduction of asthma exacerbation frequency is an important measure in asthma studies, and fewer exacerbations indicate better asthma control and reduced morbidity and mortality. Ideally, treatments should stabilize symptoms for long periods and prevent exacerbations.

The frequency of acute exacerbations was evaluated in two studies (long-term efficacy) with very low-quality evidence (Table 3). The results showed AAHM plus RP was not better than placebo plus RP in reducing the frequency of acute exacerbations, and the heterogeneity was low (MD $-0.30,95 \%$ CI -0.93 to $-0.32, I^{2}=50 \%$; Figure 3 ).

3.5.3. Asthma Control Test. The ongoing assessment of patients with asthma is important, and symptom control is the goal of management. ACT was used to assess asthma control [34]. The ACT includes five items to assess shortness of breath, night-time waking, activity, rescue bronchodilator use, and rating of asthma control. Each item represents one question, including five answers, with a total of 25 points for all items [35]. A higher score indicates more controlled asthma.

Very low-quality evidence from two studies (171 participants) assessed the ACT (Table 3). The result of longterm efficacy showed no potential increase in ACT score (MD 1.03 points, $95 \%$ CI -0.47 to 2.53 ) when participants received AAHM plus RP, compared with those receiving placebo plus RP, and the heterogeneity was high $I^{2}=83 \%$ (Figure 4).

3.5.4. Asthma Quality of Life Questionnaire. Moderate-quality evidence from one RCT (70 participants) evaluated AQLQ (Table 3), and the result of long-term efficacy indicated that AAHM plus RP was superior to placebo plus RP in terms of improving AQLQ score (MD 6.53 points, 95\% CI 2.70 to 10.36; Figure 5).

3.5.5. Immunological Marker Total IgE. IgE is an immunological biomarker that plays an integral role in the pathogenesis of allergic diseases, including asthma [36]. The measurement of serum IgE is available in routine clinical practice and is used to define the biomarker status of patients with asthma.

Two studies (208 participants) with very low-quality evidence reported the total IgE when AAHM plus RP was compared to placebo plus RP (Table 3). The result of longterm efficacy showed treatment group was not superior to the control group at decreasing total IgE (Figure S4).

3.5.6. Eosinophil. Low-quality evidence from 1 RCT with 120 participants assessed EOS (Table 3 ). The result of longterm efficacy showed that AAHM plus RP was better than placebo plus RP (MD $-0.31,95 \%$ CI -0.37 to -0.25 ; Figure S5).

\subsection{Results of AAHM versus Placebo}

3.6.1. Lung Function. $\mathrm{FEV}_{1}$ (L) was assessed in two studies (173 participants) that were judged as low-quality evidence (Tables 5 and 6). Results showed that there was a significant difference between the groups, whether the long- or shortterm efficacy (MD 0.35 litres, 95\% CI 0.03 to 0.67 ; MD 0.66 litres, $95 \%$ CI 0.59 to 0.73 , respectively; Figure S2). Very low-quality evidence from 2 studies including 238 participants (Table 6), assessed the short-term efficacy of FEV ${ }_{1}(\%)$. The meta-analysis result indicated that there was no difference between AAHM and placebo (MD 2.20\%, 95\% CI -15.88 to $20.27, I^{2}=89 \%$; Figure 2 ).

Low-quality evidence from one study evaluated the longterm efficacy in improving the $\mathrm{FEV}_{1} / \mathrm{FVC}$ (Table 5), and the forest plot showed AAHM was better than placebo (MD $11.75 \%, 95 \%$ CI 4.04 to 19.46 ). Very low-quality evidence from two RCTs evaluated $\mathrm{FEV}_{1} / \mathrm{FVC}$ (Table 6), and the result of short-term efficacy indicated that AAHM was not superior to placebo (MD $0.68 \%, 95 \%$ CI -15.24 to 16.60 ; Figure S3).

3.6.2. Frequency of Acute Exacerbations. The frequency of acute exacerbations was assessed in 3 studies (287 participants). The results of long-term efficacy showed AAHM was better than placebo in reducing the acute exacerbations, and the quality of evidence was very low (MD -1.52, 95\% CI -2.17 to $-0.87, I^{2}=81 \%$; Table 5 and Figure 3 ). Another short-term efficacy observation with moderate evidence showed that AAHM was also superior to placebo (MD -1.84, 95\% CI -2.32 to -1.36 ; Table 6 and Figure 3 ).

3.6.3. Asthma Quality of Life Questionnaire. Moderate-quality evidence from 2 studies (238 participants) assessed the AQLQ (Table 6). The result of short-term efficacy showed a potential increase in AQLQ score (MD 6.57 points, 95\% CI 3.76 to 9.38) when participants received AAHM, compared with those receiving placebo alone, and the heterogeneity was low $I^{2}=0 \%$ (Figure 5).

3.6.4. Immunological Marker Total IgE. Low-quality evidence from 1 RCT with 120 participants assessed total IgE (Table 6). The result of short-term efficacy showed the total 


\begin{tabular}{|c|c|c|c|c|c|c|c|c|c|c|c|c|}
\hline \multirow[b]{2}{*}{ Study or Subgroup } & \multicolumn{3}{|c|}{ Treatment group } & \multicolumn{3}{|c|}{ Control group } & \multirow[b]{2}{*}{ Weight } & \multirow{2}{*}{$\begin{array}{c}\text { Mean Difference } \\
\text { IV, Random, 95\% CI }\end{array}$} & \multirow{2}{*}{\multicolumn{4}{|c|}{$\begin{array}{l}\text { Mean Difference } \\
\text { IV, Random, 95\% CI }\end{array}$}} \\
\hline & Mean & SD & Total & Mean & SD & Total & & & & & & \\
\hline \multicolumn{13}{|c|}{ 3.4.1 Long-term efficacy: AAHM plus RP vs Placebo plus RP } \\
\hline Wang 2016 [19] & 2.42 & 0.41 & 44 & 2.53 & 0.42 & 44 & $21.2 \%$ & $-0.11[-0.28,0.06]$ & & - & & \\
\hline Yang 2018 [15] & 2.7 & 1.41 & 30 & 3.53 & 2.35 & 30 & $17.4 \%$ & $-0.83[-1.81,1.15]$ & & & & \\
\hline \multirow{2}{*}{\multicolumn{13}{|c|}{$\begin{array}{l}\text { Heterogeneity: } \mathrm{Tau}^{2}=0.13 ; \mathrm{Chi}^{2}=2.01, \mathrm{df}=1(P=0.16) ; I^{2}=50 \% \\
\text { Test for overall effect: } Z=0.95(P=0.34) \\
\text { 3.4.2 Long-term efficacy: AAHM vs Placebo }\end{array}$}} \\
\hline & & & & & & & & & & & & \\
\hline Bai $2010[26]$ & 2.31 & 0.869 & 42 & 3.439 & 1.598 & 42 & $19.9 \%$ & $-1.13[-1.68,-0.58]$ & & - & & \\
\hline Liu 2018 [22] & 1.9 & 0.3 & 30 & 3.7 & 0.4 & 30 & $21.2 \%$ & $-1.80[-1.98,-1.62]$ & 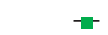 & & & \\
\hline Subtotal $(95 \% \mathrm{CI})$ & & & 72 & & & 72 & $41.1 \%$ & $-1.52[-2.17,-0.87]$ & & & & \\
\hline \multirow{7}{*}{\multicolumn{13}{|c|}{ 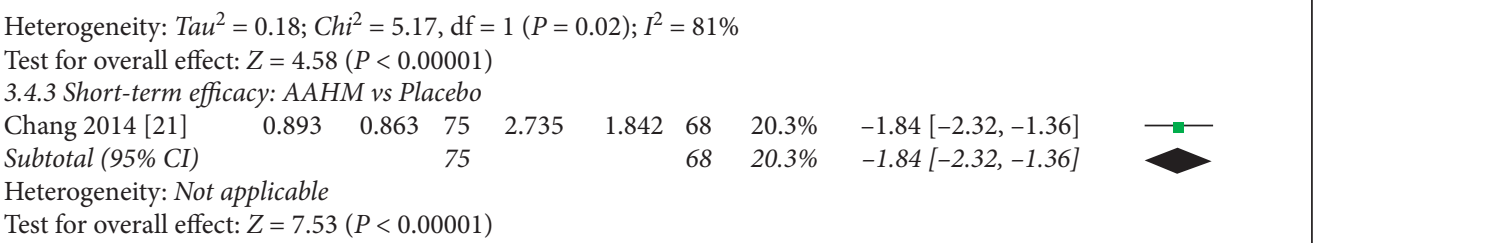 }} \\
\hline & & & & & & & & & & & & \\
\hline & & & & & & & & & & & & \\
\hline & & & & & & & & & & & & \\
\hline & & & & & & & & & & & & \\
\hline & & & & & & & & & & & & \\
\hline & & & & & & & & & & & & \\
\hline & & & & & & & & & -2 & -1 & 1 & 2 \\
\hline & & & & & & & & & Treatmen & nt group & Control \& & group \\
\hline
\end{tabular}

FIGURE 3: Forest plot of treatment group versus control group: frequency of acute exacerbations.

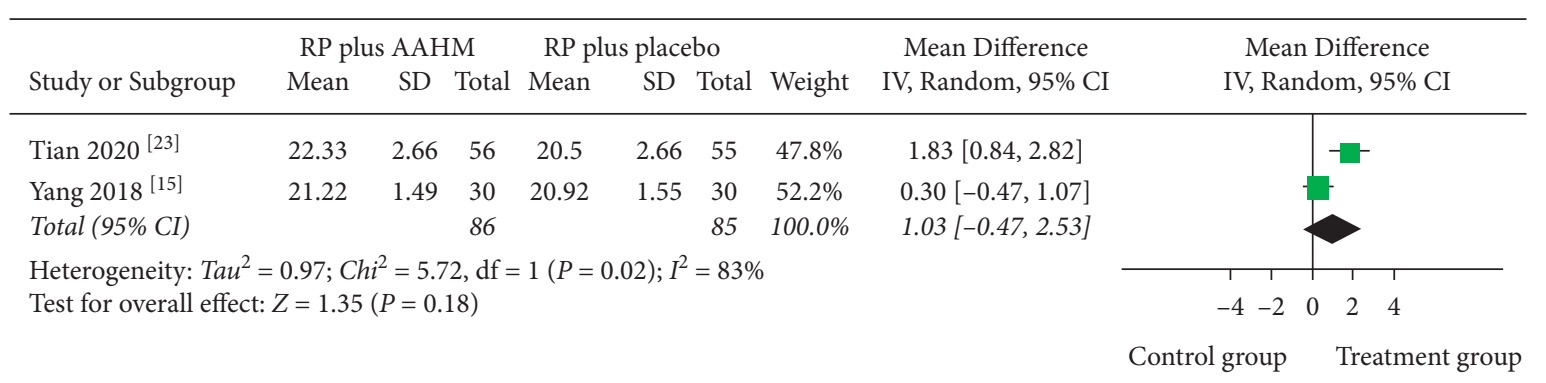

FIGURE 4: Forest plot of treatment group versus control group: ACT score.

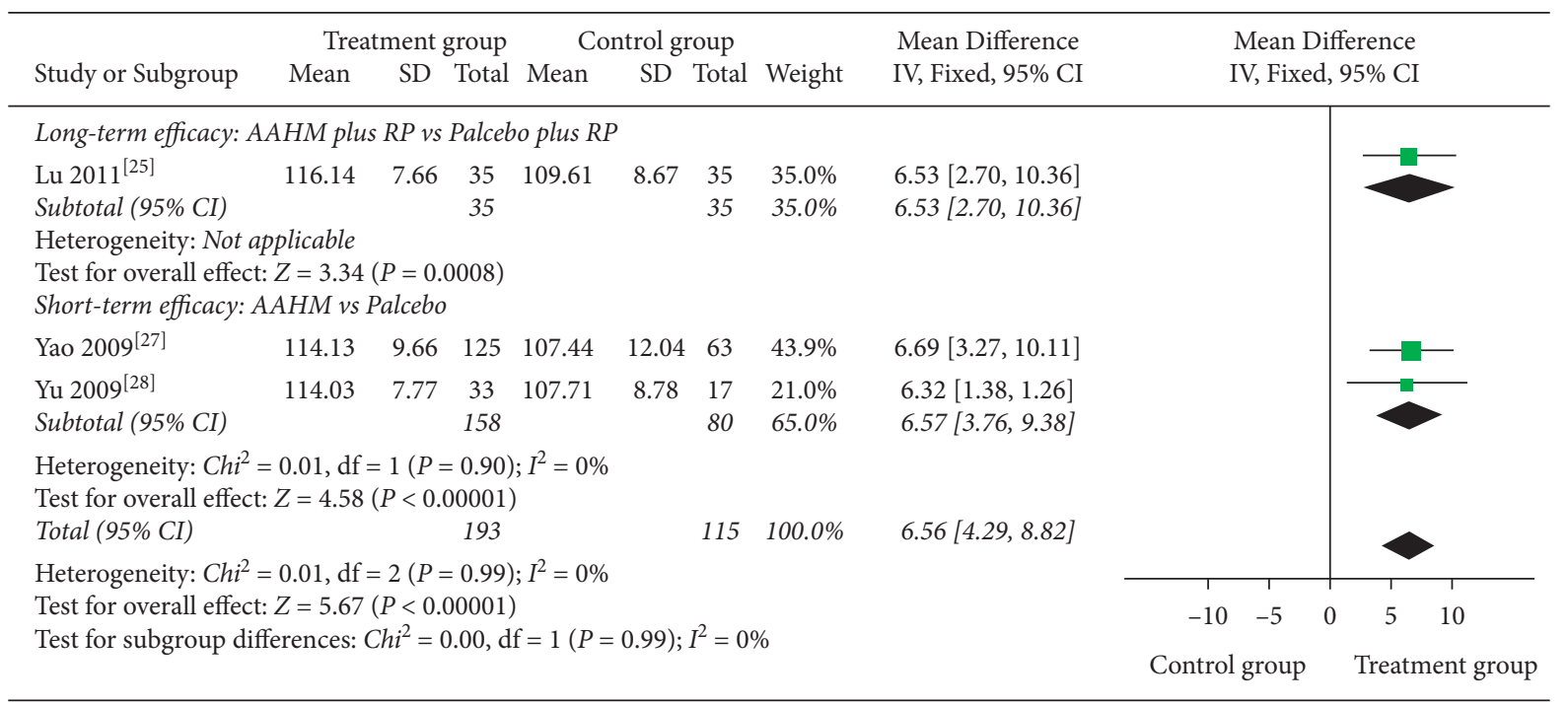

FIGURE 5: Forest plot of treatment group versus control group: AQLQ score. 
TABLE 5: Summary of GRADE evidence: comparison of long-term efficacy between AAHM and placebo

\begin{tabular}{|c|c|c|c|c|}
\hline \multirow[b]{2}{*}{ Outcomes } & \multirow[b]{2}{*}{$\begin{array}{c}\text { Number of participants (number of } \\
\text { Studies) }\end{array}$} & \multirow[b]{2}{*}{$\begin{array}{l}\text { Quality of evidence } \\
\text { (GRADE) }\end{array}$} & \multicolumn{2}{|r|}{ Absolute effect } \\
\hline & & & $\begin{array}{l}\text { Control group } \\
\text { (mean) }\end{array}$ & $\begin{array}{c}\text { Comparison between groups ( } 95 \% \\
\text { CI ) }\end{array}$ \\
\hline $\mathrm{FEV}_{1}(\mathrm{~L})$ & 66 (1 RCT) & $\oplus \oplus \bigcirc \bigcirc$ Low 3,4 & $1.64 \mathrm{~L}$ & $\uparrow 0.35(0.03,0.67)$ \\
\hline $\begin{array}{l}\mathrm{FEV}_{1} / \\
\mathrm{FVC}\end{array}$ & $92(1 \mathrm{RCT})$ & $\oplus \oplus \bigcirc \bigcirc$ Low 2,3 & $79.86 \%$ & $\uparrow 11.75(4.04,19.46)$ \\
\hline FAE & 144 (2 RCTs) & $\oplus \bigcirc \bigcirc \bigcirc$ Very Low $1,3,4$ & 3.57 times & $\downarrow-1.52(-2.17,-0.87)$ \\
\hline EOS & $92(1 \mathrm{RCT})$ & $\oplus \oplus \bigcirc \bigcirc$ Low 2,3 & $0.76 \times 0.09 / \mathrm{L}$ & No statistical significance \\
\hline
\end{tabular}

Abbreviations: AQLQ: Asthma quality of life questionnaire; FAE: Frequency of acute exacerbations; IgE: Immunoglobulin E; EOS: Eosinophil; FEV ${ }_{1}$ : Forced expiratory volume in 1s; FVC: forced vital capacity. 1. Considerable statistical heterogeneity; 2 . The credible interval contains invalid values and wide interval limits the accuracy of the results; 3 . Insufficient sample size limits the accuracy of the results; 4 . Unclear sequence generation.

TABLE 6: Summary of GRADE evidence: comparison of short-term efficacy between AAHM and placebo

\begin{tabular}{|c|c|c|c|c|}
\hline \multirow[b]{2}{*}{ Outcomes } & \multirow[b]{2}{*}{$\begin{array}{c}\text { Number of participants (number of } \\
\text { studies) }\end{array}$} & \multirow[b]{2}{*}{$\begin{array}{l}\text { Quality of evidence } \\
\text { (GRADE) }\end{array}$} & \multicolumn{2}{|r|}{ Absolute effect } \\
\hline & & & $\begin{array}{c}\text { Control group } \\
\text { (mean) }\end{array}$ & $\begin{array}{c}\text { Comparison between groups ( } 95 \% \\
\text { CI ) }\end{array}$ \\
\hline $\mathrm{FEV}_{1}(\%)$ & 238 (2 RCTs) & $\oplus \bigcirc \bigcirc \bigcirc$ Very low $1,2,3$ & $78.55 \%$ & No statistical significance \\
\hline $\mathrm{FEV}_{1}(\mathrm{~L})$ & 107 (1 RCT) & $\oplus \oplus \bigcirc \bigcirc$ Low 3,4 & $1.46 \mathrm{~L}$ & $\uparrow 0.66(0.59,0.73)$ \\
\hline $\begin{array}{l}\mathrm{FEV}_{1} / \\
\mathrm{FVC}\end{array}$ & 238 (2 RCTs) & $\oplus \bigcirc \bigcirc \bigcirc$ Very low $1,2,3$ & $71.07 \%$ & No statistical significance \\
\hline FAE & $143(1 \mathrm{RCT})$ & $\oplus \oplus \oplus \bigcirc$ Moderate 3 & 2.74 times & $\downarrow-1.29(-1.66,-0.93)$ \\
\hline AQLQ & $238(2 \mathrm{RCT})$ & $\oplus \oplus \oplus \bigcirc$ Moderate 3 & 107.58 points & $\uparrow 6.57(3.76,9.38)$ \\
\hline Total IgE & 120 (1 RCT) & $\oplus \oplus \bigcirc \bigcirc$ Low 3,4 & $208.50 \mathrm{IU} / \mathrm{ml}$ & $\downarrow-104.40(-113.14,-95.66)$ \\
\hline EOS & 66 (1 RCT) & $\oplus \oplus \bigcirc \bigcirc$ Low 2,3 & $0.31 \times 109 / \mathrm{L}$ & No statistical significance \\
\hline
\end{tabular}

Abbreviations: AQLQ: asthma quality of life questionnaire, FAE: frequency of acute exacerbations, IgE: immunoglobulin E, EOS: eosinophil, FEV ${ }_{1}$ : forced expiratory volume in 1 second, and FVC: forced vital capacity. 1. Considerable statistical heterogeneity, 2. the credible interval contains invalid values and wide interval limits the accuracy of the results, 3. insufficient sample size limits the accuracy of the results, and 4. unclear sequence generation.

IgE reduced in participants receiving AAHM compared to those receiving placebo (MD - $104.40 \mathrm{IU} / \mathrm{ml}, 95 \% \mathrm{CI}-113.14$ to -95.66 ; Figure $\mathrm{S} 4)$.

3.6.5. Eosinophil. Low-quality evidence from two RCTs evaluated EOS (Tables 5 and 6). The result indicated that there was no difference between AAHM and placebo, whether the long- or short-term efficacy (Figure S5).

3.7. Adverse Events. The occurrence of adverse events was reported in three studies $[15,21,23]$, and no serious adverse events were reported. These studies included 13 participants and reported adverse events associated with acupoint sticking. Adverse events associated with AAHM were judged to be of mild intensity and consisted of local skin redness, swelling, itching, or blisters. These symptoms were related to direct irritation caused by the herbal medicine and duration of acupoint application. Symptoms were relieved by reducing the acupoint application time, maintaining dryness, or coating the affected area with mupirocin antibiotic ointment.

\section{Discussion}

Asthma is an increasingly prevalent health problem worldwide; the prevalence of asthma among people aged 20 and over in China is $4.2 \%$, with 45.7 million patients [1, 37].
AAHM is the topical application of Chinese herbal paste on acupuncture points. AAHM therapy for asthma has received widespread research attention over the past 30 years. Nowadays, AAHM as a complementary and alternative therapy has been widely used for asthma in East Asian countries, including China [38, 39]. AAHM not only has the effect of stimulating acupuncture points; the active ingredients of the herbs can be absorbed through the skin. It is relatively safe and easy to apply and has a dual therapeutic effect. It is also an external therapy that does not require oral or intravenous medication. Furthermore, it is popular with both adults and children.

The primary objectives of asthma management are to control symptoms, maintain lung function and activity levels, and prevent asthma exacerbations and mortality. Two systematic reviews reported positive therapeutic effects of AAHM for asthma. They suggest that it may contribute to improving pulmonary function, clinical symptoms and reducing interleukin and IgE levels [10, 40]. However, the authors found that not enough evidence was available to conclude whether or not AAHM should be recommended. They also suggested that further research is conducted to clarify the role of AAHM.

To our knowledge, this is the first meta-analysis of AAHM versus placebo in the treatment of asthma. It is different from previous systematic reviews because it evaluates randomized double-blind placebo-controlled trials and outcomes including lung function $\mathrm{FEV}_{1}, \mathrm{AQLQ}, \mathrm{ACT}$, 
acute exacerbations, total IgE, and EOS. Sixteen studies were included for this meta-analysis. Participants receiving RP plus AAHM or AAHM alone were compared with those receiving RP plus placebo or placebo alone. Moderatequality evidence demonstrates that RP combined with AAHM or AAHM alone can improve the quality of life of asthma patients, and short-term use of AAHM was associated with a lower frequency of acute exacerbations. Moreover, very-low- to low-quality evidence also indicated that AAHM was associated with improved lung function and reduced total immunoglobulin $\mathrm{E}$ and EOS.

Chronic inflammation of the airways is the key feature of asthma. The inflammatory process is complex and involves many cells, such as eosinophils, T-lymphocytes, Mast cells, and macrophages [41]. Airway inflammation persists leading to airway damage and respiratory insufficiency, affecting the quality of life, sometimes even causing death. Exposure to environmental factors such as allergens and infections leads to asthma symptoms and morphological changes. The mechanisms of AAHM for asthma have been reported in several studies. Studies have shown that AAHM can significantly reduce asthmatic inflammation by reducing IgE, eosinophils, interleukin 4 (IL-4), and $\mathrm{CD}^{+}$levels, increasing IL-10 and $\mathrm{CD}^{+}$, and transforming growth factor beta (TGF$\beta)$ levels $[42,43]$. Results of this meta-analysis also show that AAHM has the potential in reducing total IgE and EOS. This suggests that AAHM may improve clinical symptoms by reducing airway hyper-responsiveness and regulating immune function, thus more effectively controlling asthma.

In this review, TCM prescription for acupoint application was a critical factor. The most common points across included studies were Feishu (BL13), Dingchuan (EX-B1), Tiantu (CV22), and Shenshu (BL23). TCM theory suggests that these points tonify the lungs and kidneys, thus preventing asthma. The most commonly used herbs included Xi Xin (Herba Asari), Bai Jie Zi (Semen Brassicae), Gan Sui (Radix Kansui), and Yan $\mathrm{Hu}$ Suo (Rhizoma Corydalis). According to TCM theory, these herbs can relieve cough, eliminate phlegm, and have antiasthmatic properties. Additionally, they are pungent and warm in nature, which have the characteristics of diverging and dissipating, causing them to be more easily absorbed through acupoints. These herbs can modulate humoral and cellular immune responses and reduce inflammation [44-46].

In the United States and Canada, the marketing of Xi Xin (Herba Asari) containing drugs is restricted due to aristolochic acid toxicity $[47,48]$. In China, Xi Xin is a commonly used remedy for treating colds, cough, and asthma, and there is a strict limit on the amount used in treatments. According to Chinese pharmacopoeia [49], a safe daily dose of oral Xi Xin is 1 to $3 \mathrm{~g} / \mathrm{d}$. Nevertheless, overdose can cause serious adverse effects. Another herb commonly used in the studies was Ma Huang (Herba Ephedra), which is restricted in some countries such as Australia but not in other countries such as Japan, Korea, and China, where it is widely used.

It should be noted that despite its popularity in China, Chinese medicine practitioners in other countries do not frequently use acupoints application. This may be due to the commonly observed redness, swelling, and blistering around the acupoint area. In TCM theory, AAHM therapies use herbs with warm or hot properties to irritate the skin. After the continuous stimulation of the herbs, the skin shows redness, heat, and blisters. This is why the most commonly reported adverse event in AAHM studies was skin irritation. However, this is not always considered to be an adverse effect, rather a therapeutic benefit. With this in mind, the safety of AAHM should be cautiously considered. Most of the included studies did not report whether or not adverse events had occurred and studies that did report adverse events judged them to be mild. Therefore, it is unclear the extent of AAHM's adverse event profile and interpretation of adverse events may differ based on region or country of use.

Although this review reveals the positive effects of AAHM for asthma, there remain several limitations that have some impact on the findings. Firstly, the methodology and quality of evidence of the included randomized placebocontrolled trials are still low. Almost half of the included studies did not explicitly report the method of random sequence generation, and the majority of studies did not mention allocation concealment. The results of the GRADE evaluation are mainly very-low- to low-quality evidence. Secondly, the sample sizes of the included studies were relatively small, and power calculations were not performed for the primary outcomes. Finally, most of the included studies did not report adverse events, and there remains insufficient evidence to support the safety of AAHM.

\section{Conclusion}

Evidence from this meta-analysis suggests that AAHM may be an effective treatment for asthma, and it can be performed as an alternative therapy for asthma. AAHM shows great potential in improving the quality of life, improving lung function, and reducing acute exacerbation of asthma. However, the current evidence is not strong enough to routinely recommend AAHM for patients with asthma. The benefits and safety of AAHM still need further evaluation due to study design and reporting weaknesses.

\section{Abbreviations}

95\% CI: $\quad$ 95\% Confidence interval

AAHM: Acupoint application of herbal medicine

ACT: Asthma control test

AMED: $\quad$ Allied and Complementary Medicine Database

AQLQ: Asthma quality of life questionnaire

CBM: $\quad$ Chinese Biomedical Database

CINAHL: Cumulative Index of Nursing and Allied Health Literature

CENTRAL: Cochrane Central Register of Controlled Trials

CNKI: China National Knowledge Infrastructure

CQVIP: ChongQing VIP

Embase: Excerpta Medica Database

$\mathrm{FEV}_{1}$ : $\quad$ Forced expiratory volume in 1 second

FVC: $\quad$ Forced vital capacity

GINA: Global Initiative for Asthma

IgE: Immunoglobulin $\mathrm{E}$ 


$\begin{array}{ll}\text { MCID: } & \text { Minimal clinically important difference } \\ \text { MD: } & \text { Mean difference } \\ \text { PEF: } & \text { PEAK expiratory flow } \\ \text { RCTs: } & \text { Randomized controlled trials } \\ \text { RevMan: } & \text { Review Manager } \\ \text { RP: } & \text { Routine pharmacotherapy } \\ \text { TCM: } & \text { Traditional Chinese medicine } \\ \text { WFMO: } & \text { Wanfang Medicine Online. }\end{array}$

\section{Data Availability}

All data of this review are available from the public, openaccess electronic databases.

\section{Conflicts of Interest}

The authors declare that there are no conflicts of interest and are responsible for the content and writing of the paper.

\section{Authors' Contributions}

ZHW, YCZ, and YXC contributed equally. YJX and YBC conceived the project. ZHW contributed to analyzing the data and drafting the manuscript. YCZ, YXC, and $\mathrm{BH}$ contributed to searching the electronic databases, screening, and data extraction. YCZ, YXC, and LW evaluated the risk of bias and interpreted the evidence from a clinical perspective. YJX, YBC, and LL revised the contents and polished the language of the translation. All authors read and approved the final manuscript. The authors thanked Han Xie for her excellent work on this study.

\section{Acknowledgments}

This research was supported by the State Key Laboratory of Dampness Syndrome of Chinese Medicine (Grant no. SZ2021ZZ42), Science and Technology Planning Project of Guangzhou (Grant no. 202002020042), Guangdong Provincial Science and Technology Innovation Strategy Special Fund (Guangdong-Hong Kong-Macau Joint Lab, Grant no. 2020B1212030006), double first-class and high-level university discipline collaborative innovation team project of Guangzhou University of Chinese Medicine (Grant nos. 2021XK10 and 2021XK27), Scientific Research Project of Guangdong Provincial Bureau of Chinese Medicine (Grant no. 20221175), and Zhongying Zhou Famous Doctors' Workshop of Guangdong Provincial Hospital of Chinese Medicine.

\section{Supplementary Materials}

Figure S1. Risk of bias graph. Figure S2. Forest plot of treatment group versus control group: $\mathrm{FEV}_{1}(\mathrm{~L})$. Figure S3. Forest plot of treatment group versus control group: $\mathrm{FEV}_{1} /$ FVC. Figure S4. Forest plot of treatment group versus control group: total IgE. Figure S5. Forest plot of treatment group versus control group: EOS $\left(\times 10^{9} / \mathrm{L}\right)$. (Supplementary Materials)

\section{References}

[1] M. Masoli, D. Fabian, S. Holt, and R. Beasley, "The global burden of asthma: executive summary of the GINA Dissemination Committee Report," Allergy, vol. 59, no. 5, pp. 469-478, 2004.

[2] N. G. Papadopoulos, H. Arakawa, K.-H. Carlsen et al., "International consensus on (ICON) pediatric asthma," Allergy, vol. 67, no. 8, pp. 976-997, 2012.

[3] L. J. Akinbami, J. E. Moorman, and P. L. Garbe, "Status of childhood asthma in the United States, 1980-2007," Pediatrics, vol. 123, 2009.

[4] K.-L. Hon, C. Fung, A. Leung, T. Leung, and D. Ng, "Complementary and alternative medicine for childhood asthma: an overview of evidence and patents," Recent Patents on Inflammation \& Allergy Drug Discovery, vol. 9, no. 1, pp. 66-79, 2015.

[5] Global Strategy for Asthma Management and Prevention, "Global initiative for asthma (GINA)," 2013, http://www. ginasthma.org/.

[6] G. Passalacqua, M. Albano, G. W. Canonica et al., "Inhaled and nasal corticosteroids: safety aspects*," Allergy, vol. 55, no. 1, pp. 16-33, 2000.

[7] X. X. Xiang, J. Y. Zhang, and Y. M. Si, "Study on the mechanism of acupoint sticking therapy with Chuan fuling for treatment of asthma model rats," Chinese Acupuncture \& Moxibustion, vol. 29, p. 739, 2009.

[8] L. Hb, "The Clinical effect of acupoint application to asthma rats and the impact to IL-4," Journal of Southern Medical University, vol. 29, pp. 565-567, 2009.

[9] Diagnosis and treatment guidance of Asthma, "Diagnosis and treatment guidance of Asthma," Chinese Journal of Tuberculosis and Respiratory Diseases, vol. 39, pp. 1-24, 2016.

[10] S. H. Lee, G. T. Chang, X. Zhang, and H Lee, "Acupoint herbal patching for asthma: a systematic review and meta-analysis of randomized controlled trials," Medicine, vol. 95, p. e2439, 2016.

[11] Global Strategy for Asthma Management and Prevention, "Global initiative for asthma (GINA)," 2015, http://www. ginasthma.org/.

[12] Chinese Medical Association of respiratory disease branch Asthma Study Group, "Guidelines for the prevention and treatment of bronchial asthma (Definition, diagnosis, treatment, education and management of bronchial asthma)," Chinese Journal of Tuberculosis and Respiratory Diseases, vol. 26, pp. 132-138, 2003.

[13] J. P. T. Higgins and S. Green, Cochrane Handbook for Systematic Reviews of Interventions, http://training.cochrane.org/ handbook (updated 2016)," Cochrane Collaboration website.

[14] H. Schunemann, J. Brozek, G. Guyatt et al., "GRADE handbook for grading quality of evidence and strength of recommendations (The GRADE Working Group)," Available from, 2013

[15] L. Yang, Clinical Observation on the Prevention of Bronchial Asthma Attack by the Acupoint Application of Summer Treatment of Winter Disease, Hubei University of Chinese Medicine, 2018.

[16] E. J. Wang and Z. X. Zhang, "Effect of point application of Fujiutian on lung function, Ig E and EOS in children with remission stage of bronchial asthma," Journal of New Chinese Medicine, vol. 49, pp. 110-113, 2017.

[17] H. M. Wang, "Clinical observation on 50 cases of child bronchial asthma treated by acupoint application of Fujiutian 
combined with Montelukast," Chinese Journal of Ethnomedicine and Ethnopharmacy, vol. 26, pp. 83-85, 2017.

[18] C. Ma, "Impact of winter diseases treated with acupoints sticking therapy in summer on immune cytokines and pulmonary function of children with bronchial asthma at remittent stage," Practical Journal of Cardiac Cerebral Pneumal and Vascular Disease, vol. 24, pp. 91-94, 2016.

[19] Y. L. Wang and F. Wang, "Clinical observation on the prevention of bronchial asthma during non-acute attack treated by acupoint application of Dog Days," Chinese Journal of Lung Diseases, vol. 9, pp. 317-319, 2016.

[20] L. J. Shi and Y. Zhao, "Clinical observation on prevention of 92 children with asthma recurrence by the acupoint application of summer treatment of winter disease," Journal of Emergency in Traditional Chinese Medicine, vol. 23, pp. 2342-2344, 2014.

[21] J. J. Chang and S. L. Ma, "Clinical research on effect of acupoint sticking in prevention of bronchial asthma," Research of Integrated Traditional Chinese and Western Medicine, vol. 6, pp. 131-132+136, 2014.

[22] C. Y. Liu, W. Z. Wu, and Y. G. Fang, "Clinical research on the prevention and treatment of bronchial asthma by acupoint application of baijiezi tufan," Journal of Nanjing University of Traditional Chinese Medicine, vol. 34, pp. 565-568, 2018.

[23] X. Tian, Clinical Observation on Treatment of Chronic Persistent Asthma with Lung Spleen Qi Deficiency Syndrome by Acupoint Application Based on Syndrome Differentiation, Liaoning University of Traditional Chinese Medicine, Liaoning, China, 2020.

[24] W. J. Shangguan and H. Li, "The effects of penetration along meridians therapy on $\mathrm{CD}^{+}, \mathrm{CD}^{+}$, Regulatory $\mathrm{T}$ Cells and quality of life in asthmatic patients," Journal of Shandong University of Traditional Chinese Medicine, vol. 37, pp. 125-126, 2013.

[25] Y. M. Lu, "A summary of 35 cases of bronchial asthma in remission stage treated by acupoint application of Dog Days," Hunan Journal of Traditional Chinese Medicine, vol. 27, pp. 17-19, 2011.

[26] J. M. Bai and A. F. Yang, "Clinical research on the treatment of bronchial asthma treated by acupoint application of Dog Days," Journal of Shanxi College of Traditional Chinese Medicine, vol. 11, pp. 51-53, 2010.

[27] H. Yao, J. Tong, and P. D. Zhang, "Acupoint sticking therapy for treatment of bronchial asthma: a multicenter controlled randomized clinical trial," Chinese Vesiculation and Moxibustion, vol. 29, pp. 609-612, 2009.

[28] D. D. Yu and H. Yao, "Clinical study of bronchial asthma treated with acupoint application therapy," Academic Journal of Guangzhou, vol. 37, pp. 21-24, 2009.

[29] H. Yao, J. Tong, and P. D. Zhang, "The effects of acupoint application therapy of traditional Chinese medicine on inflammatory cell level in patients with bronchial asthma," Journal of Guangzhou College of Traditional Chinese Medicine, pp. 289-292, 2007.

[30] H. P. Hu, F. Luo, and J. Y. Zhang, "Clinical observation on 181 cases of bronchial asthma treated by crude herb Moxibustion," Journal of New Chinese Medicine, pp. 61-62, 2005.

[31] Chinese Medical Association of respiratory disease branch Asthma Study Group, "Prevention and treatment of asthma guideline," Chinese Journal of Tuberculosis and Respiratory Diseases, vol. 20, pp. 261-267, 1997.

[32] H. Schünemann, J. Brozek, and A. Oxman, GRADE Handbook for Grading Quality of Evidence and Strength of
Recommendations, Updated October 2013. The GRADE Working Group,Available from, 2013.

[33] H. K. Reddel, D. R. Taylor, E. D. Bateman et al., "An official American thoracic society/European respiratory society statement: asthma control and exacerbations," American Journal of Respiratory and Critical Care Medicine, vol. 180, no. 1, pp. 59-99, 2009.

[34] R. A. Nathan, C. A. Sorkness, M. Kosinski et al., "Development of the asthma control test污A survey for assessing asthma control," The Journal of Allergy and Clinical Immunology, vol. 113, no. 1, pp. 59-65, 2004.

[35] M. Schatz, C. A. Sorkness, J. T. Li et al., "Asthma Control Test: reliability, validity, and responsiveness in patients not previously followed by asthma specialists," The Journal of Allergy and Clinical Immunology, vol. 117, no. 3, pp. 549-556, 2006.

[36] D. B. Corry and F. Kheradmand, "Induction and regulation of the IgE response," Nature, vol. 402, no. S6760, pp. 18-23, 1999.

[37] K. Huang, T. Yang, J. Xu et al., "Prevalence, risk factors, and management of asthma in China: a national cross-sectional study," The Lancet, vol. 394, no. 10196, pp. 407-418, 2019.

[38] Beijing Chinese Medicine Information Nets, "The list of Sanfu acupoint application therapy service agencies in Beijing in 2014," 2014.

[39] W. Cai, “Taiwanese excited about Sanfu patches," 2014, http:// www.huaxia.com/jitw/dnsh/2010/07/1994062.html.

[40] L. Su, L. Meng, R. Chen, W. Wu, B. Peng, and L. Man, "Acupoint Application for asthma therapy in adults: a systematic review and meta-analysis of randomized controlled trials," Complementary Medicine Research, vol. 23, no. 1, pp. 16-21, 2016.

[41] W. W. Busse and R. F. Lemanske, “Asthma," New England Journal of Medicine, vol. 344, no. 5, pp. 350-362, 2001.

[42] H. U. Xiaoling, "Influence of three nine acupoint application on serum ige in asthmatic rats," Journal of Xinjiang Medical University, vol. 24, 2013.

[43] Y. M. Zhang, "Effect of acupoint application on eos in blood and bronchial tissue of asthma model guinea pigs," Journal of Traditional Chinese Medicine University of Hunan, vol. 43, 2006.

[44] S. H. Cin, "The research situation of Baijiezi Acupoint Application," Modern J Integr Tradit Chin West Med, vol. 23, pp. 3181-3184, 2014.

[45] Y. P. Wu, "A comparison of anti-asthmatic effects of Baijiezi tufa from compound Chinese traditional medicine and single Chinese herb on Guinea pigs," Jiangsu Medical Journal, vol. 37, pp. 1643-1645+1608, 2011.

[46] G. Fu, "Study on the basic herbal medicines and acupoints in the therapy of acupoint Application for winter-diseasetreated-in-summer," Journal of Traditional Chinese Medicine, vol. 50, pp. 794-797, 2009.

[47] U.S. Food and Drug Administration, Department of Health \& Human Services. FDA Poisonous Plant Database, U.S. Food and Drug Administration, Newyork, NY, USAhttps://www. accessdata.fda.gov/scripts/plantox/detail.cfm?id=7789.

[48] A. K. Drew, I. M. Whyte, A. Bensoussan, A. H. Dawson, X. Zhu, and S. P. Myers, "Chinese herbal medicine toxicology database: monograph on herba Asari, "Xi Xin"," Journal of Toxicology - Clinical Toxicology, vol. 40, no. 2, pp. 169-172, 2002.

[49] Chinese Pharmacopoeia Commission, Pharmacopoeia of the People's Republic of China, People's Medical Publishing House, Beijing, 2005. 